\title{
Prostate artery embolization has long term efficacy for treatment of severe lower urinary tract symptoms from giant prostatic hyperplasia
}

\author{
Alexander S. Somwaru ${ }^{1 *} \mathbb{0}$, Stephen Metting ${ }^{2}$, Laura M. Flisnik ${ }^{3}$, Michael G. Nellamattathil ${ }^{4}$, Arjun Sharma ${ }^{5}$ \\ and Venkat S. Katabathina ${ }^{6}$
}

\begin{abstract}
Background: Patients with severe lower urinary tract symptoms (LUTS) from giant prostatic hyperplasia (GPH): prostate volume greater than $200 \mathrm{~mL}$ that do not respond to medical therapy may not be eligible for surgical treatments due to morbidities, technical challenges, and patient preference. This retrospective investigation examined the long-term efficacy and safety of prostatic arterial embolization (PAE) as a treatment option for severe LUTS due to GPH in a large patient cohort.
\end{abstract}

Methods: Of 529 patients who underwent PAE between January 2016 and January 2020, 72 patients had severe LUTS from GPH and were retrospectively evaluated. PAE was performed with two embolic agents in sequence: 100-250 $\mathrm{mm}$ particles followed by $2 \mathrm{~mm}$ and $3 \mathrm{~mm}$ coils. Clinical assessment was performed with international prostate symptoms score (IPSS), quality of life (QoL), peak flow rate (Qmax), post-void residual volume (PVR), and prostate specific antigen (PSA) measurements before and 12 months and 24 months after PAE. Prostate volume (PV) was measured by multiparametric magnetic resonance (MR) imaging before and 12 months and 24 months after PAE.

Results: Patients with severe LUTS from GPH experienced significant clinical improvements in IPSS, QoL, Qmax, PVR, PSA, and PV at 12 months and 24 months after PAE. Mean IPSS decreased from 26.5 to $18.0(P<0.01)$ to $10.5(P<0.01)$. Mean QoL decreased from 6.0 to $4.0(P<0.01)$ to $2.0(P<0.01)$. Mean Qmax increased from 8.0 to $14 \mathrm{~mL} / \mathrm{s}(P<0.01)$ to $18 \mathrm{~mL} / \mathrm{s}(P<0.01)$. Mean PVR decreased from 198.0 to $152.0 \mathrm{~mL}(P<0.01)$ to $90 \mathrm{~mL}(P<0.01)$. Mean PV decreased from $303.0 \mathrm{~mL}$ to $258.0 \mathrm{~mL}(P<0.01)$ to $209.0 \mathrm{~mL}(P<0.01)$. Mean PSA decreased from $11.2 \mathrm{ng} / \mathrm{mL}$ to $9.5 \mathrm{ng} / \mathrm{mL}(P<0.05)$ to $7.9 \mathrm{ng} / \mathrm{mL}(P<0.05)$. No major complications occurred.

Conclusions: PAE is a safe treatment with long term efficacy for severe LUTS from GPH. PAE may be a viable therapeutic option for patients with severe LUTS from GPH whom fail medical therapy and are not candidates for surgical treatments.

Keywords: Giant prostatic hyperplasia (GPH), Prostatic artery embolization (PAE), Lower urinary tract symptoms (LUTS)

\footnotetext{
*Correspondence: alex.somwaru@gmail.com

${ }^{1}$ Department of Diagnostic, Molecular, and Interventional Radiology, Icahn School of Medicine At Mount Sinai, 1000 10th Avenue, New York, NY 10019, USA

Full list of author information is available at the end of the article
}

\section{Background}

Patients with severe urinary tract symptoms (LUTS) from prostatic hyperplasia that is refractory to medical therapy are offered surgical treatment options that range from minimally invasive procedures to transurethral resection of the prostate (TURP) to simple prostatectomy [1-4]. The surgical treatment selection process is

(c) The Author(s). 2020 Open Access This article is licensed under a Creative Commons Attribution 4.0 International License, which permits use, sharing, adaptation, distribution and reproduction in any medium or format, as long as you give appropriate credit to the original author(s) and the source, provide a link to the Creative Commons licence, and indicate if changes were made. The images or other third party material in this article are included in the article's Creative Commons licence, unless indicated otherwise in a credit line to the material. If material is not included in the article's Creative Commons licence and your intended use is not permitted by statutory regulation or exceeds the permitted use, you will need to obtain permission directly from the copyright holder. To view a copy of this licence, visit http://creativecommons.org/licenses/by/4.0/. The Creative Commons Public Domain Dedication waiver (http://creativeco mmons.org/publicdomain/zero/1.0/) applies to the data made available in this article, unless otherwise stated in a credit line to the data. 
governed by prostate volume, presence of LUTS, comorbidities, risk-benefit profile, patient preference treatment availability and physician expertise [1-4]. Prostate volume is a significant factor in surgical treatment selection [1-4]. TURP and minimally invasive procedures, such as photoselective vaporization, laser enucleation, transurethral vapor and microwave ablation, transurethral incision, and prostate urethral lift, are best suited for patients with prostate volumes less than $80 \mathrm{~mL}$ [1-4]. Large prostatic hyperplasia is defined as prostate volume greater than $100 \mathrm{~mL}$ and giant prostatic hyperplasia $(\mathrm{GPH})$ is defined as prostate volume greater than $200 \mathrm{~mL}$ [1-9]. Patients with large and giant prostatic hyperplasia with severe LUTS may require simple prostatectomy however may have a higher risk of morbidity from blood loss that requires blood transfusion (8-15\%), urinary incontinence $(10 \%)$, and urinary bladder neck stenosis and urethral strictures (5\%) [1-9)].

Prostate artery embolization (PAE) has emerged as a safe and efficacious treatment of patients with severe LUTS from prostatic hyperplasia with prostate volumes up to $100 \mathrm{~mL}$, or large prostatic hyperplasia [5-13]. Numerous retrospective and prospective studies of large cohorts of patients have shown that PAE safely provides long term reduction in symptoms and prostate volume by inducing ischemic necrosis, diminished hormonal response, and reduced prostate innervation with consequent reduced smooth muscle tone [10-13].

$\mathrm{GPH}$ is defined as prostate volume greater than $200 \mathrm{~mL}$ [1-9, 14-19]. GPH has been described in several individual patients as case reports in a review of international scientific literature [1-9, 14-19]. Individual cases of patients with severe LUTS from GPH whom underwent treatment with surgery or minimally invasive ablative urological treatment have also been reported [20-26]. However the safety and long-term efficacy of PAE for treatment of large cohorts of patients with severe LUTS from GPH has not been examined. One study retrospectively reviewed eight patients and one case report reviewed one patient whom underwent PAE for severe LUTS from GPH [27, 28]. While these two studies reported short term efficacy: eight months and three months, respectively, for significant symptom relief, long term efficacy remains unknown [27, 28]. A prostate volume limit that may render PAE ineffective, due to unsuccessful symptom and prostate volume reduction, has also not been established [1-4, 27, 28]. This study evaluated the safety and long-term efficacy of PAE in the largest cohort of patients, to date, with severe LUTS due to $\mathrm{GPH}$.

PAE has a steep learning curve because it requires both refined microvascular skills and detailed knowledge of highly variable anatomic anatomy to identify, catheterize, and embolize target prostatic arteries to attain successful outcomes and avoid adverse complications [5-8]. The variant origins and anatomies of the prostate artery have been, in turn, reported in a variable fashion across anatomic and angiographic literature. The most commonly reported origins are the internal pudendal artery, the superior vesical artery (with a common trunk), inferior vesical artery, the glutealpudendal arterial trunk, and the obturator artery [511]. Prostate artery anatomy origins, number, accessory branches, and extra-prostatic anastomoses of the prostate artery may vary from left to right pelvic side. The prostatic artery bifurcates into the central and capsular divisions. The superior and anterolateral pedicle, which is the primary arterial target of PAE, provides the dominant supply to the central gland and the inferior and posterolateral pedicle provides supply to the peripheral gland and prostate apex [5-12]. In hyperplastic prostate glands, the prostate artery is tortuous with intraglandular twists and spiral arterial branches, which are used as landmarks for angiographic identification and confirmation of catheter position during PAE [5-13]. Extra-arterial anastomoses commonly communicate with the internal pudendal artery, contralateral prostate artery, and superior and inferior vesical arteries [5-10, 12].

Clinical volume and experience allow the operator to develop a level of expertise in PAE, which is a technically challenging although safe and efficacious procedure, however complications may occur. PAE is performed under direct fluoroscopic guidance with cone-beam CT. Cone-beam CT is particularly useful to assist in the identification of the prostatic arteries, reduce ionizing radiation dose and procedural time, and minimize complications of nontarget embolization. Non-target embolization of pelvic organs, like the urinary bladder, urethra, rectum, and penis, is a source of complications such a hematuria, rectal bleeding, erectile dysfunction, penile skin changes and pain that usually resolve without intervention or surgery [1-5]. Acute urinary retention and dysuria may be caused increased pressure and compression of the intraprostatic urethra from swelling, edema, ischemia, and necrosis of the prostate gland that occur after embolization that may require temporary post-procedural catheterization of the urinary bladder until spontaneous urination resumes [1-5]. Urinary tract infections, also thought to be caused by increased pressure and compression of the intraprostatic urethra from swelling, edema, ischemia, and necrosis of the prostate gland, require outpatient antibiotic therapy [1-5]. Other self-limited complications include hematospermia, urethral pain, and soft tissue hematomas at the arterial access site [1-5]. 


\section{Methods}

Institutional review board (IRB) approval was received for this retrospective study. Written informed consent was obtained from the patients and any accompanying images. Patients gave consent for their personal or clinical details along with any identifying images to be published in this study. A review of the electronic medical records (EMR) from a single medical center was conducted on all patients (529) whom underwent PAE for severe LUTS between January 2016 and January 2020. Severe LUTS was classified as International Prostate Symptom Score (IPSS) greater than 18 points, urinary quality of life (QoL) greater than 3 points, and peak flow rate (Qmax) less than $12 \mathrm{~mL} / \mathrm{s}$. Patients were evaluated in this investigation if they underwent PAE for severe LUTS from GPH. GPH is defined as prostate volume greater than $200 \mathrm{~mL}$. Patients that did not have GPH (prostate volumes less than $200 \mathrm{~mL}$ ) were not evaluated in this investigation. Of the 529 patients that underwent PAE for severe LUTS, 72 patients had GPH and were evaluated in this investigation. At our institution, multiparametric MR imaging is performed on all patients before PAE for prostate volume (PV) measurements and to ensure the absence of any lesions suspicious for clinically significant cancers according to version 2.1 of Prostate Imaging-Reporting and Data System (PI-RADS) [29]. No patients underwent PAE if MR imaging revealed lesions suspicious for clinically significant cancer. MR imaging was also used to measure prostate volumes at 12 months and 24 months after PAE. Patients underwent PAE if they did not respond to 5-alpha-reductase inhibitor and/or alpha-1-adrenergic receptor antagonist medical therapy for at least 6 months or longer and were not eligible for surgery or refused surgery. Patients were not eligible for surgical treatment due to comorbidities and/or contraindications to surgery and/or general anesthesia 49 (68\%) patients with cardiovascular disease on anticoagulation/ antiplatelet therapy and $23(32 \%)$ patients with chronic lung disease. IPSS, QoL, Qmax, PV, postvoid residual volume (PVR), and PSA were measured and collected before PAE and 12 months and 24 months after PAE. Because MR imaging is performed on all patients before PAE to ensure the absence of any lesions suspicious for clinically significant cancers in our institution, patients with PSA levels above $4 \mathrm{ng} / \mathrm{mL}$ were examined in this investigation. Before PAE, 5-alpha-reductase inhibitor therapy was stopped and alpha-1-adrenergic receptor antagonist therapy was tapered and stopped after one month after PAE. No patients underwent additional interventions after PAE.

\section{Clinical metrics}

Clinical assessment was performed in all patients before PAE and 12 months and 24 months after PAE with history and physical examination, IPSS and QoL questionnaires, uroflowmetry Qmax, PVR, and PSA levels measurements. These clinical metrics are routinely recorded for patient care, to optimize patient outcomes, and for quality improvement and performance.

\section{Prostate MR imaging and volume measurement}

The protocol at our institution is to perform multiparametric MR imaging for all patients before PAE for prostate gland volume measurements and to ensure the absence of clinically significant cancers before PAE. Prostate volumes were calculated using MR imaging obtained before PAE and 12 months and 24 months after PAE. At our institution, multiparametric prostate MR imaging is performed using 3.0 T magnet systems (Siemens Healthcare, Erlangen, Germany). Exams are performed with phased array torso coils using the following protocol (Table 1): axial, sagittal, and coronal T2-weighted turbo spin echo images; axial b50, b500, and b800 s/ $\mathrm{mm}^{2}$ diffusion-weighted images; synthetic extrapolated b1200,

Table 1 MRI acquisition protocol

\begin{tabular}{|c|c|c|c|c|c|c|}
\hline Sequence & Plane & Slice/gap (mm) & TR/TE (ms) & FOV (mm) & Matrix & Flip angle \\
\hline T2WTSE & Sagittal & $5 / 1$ & $4000 / 100$ & $200 \times 200$ & $384 \times 224$ & 150 \\
\hline T2W TSE & Axial & $3 / 0$ & $4000 / 100$ & $200 \times 200$ & $384 \times 224$ & 150 \\
\hline T2W TSE & Coronal & $3 / 0$ & $4000 / 100$ & $200 \times 200$ & $384 \times 224$ & 150 \\
\hline $\begin{array}{l}\text { DWI (b0, 50, 500, 800, 1200, 1500, } \\
\left.\text { 2000, } 2500 \mathrm{~s} / \mathrm{mm}^{2}\right) / A D C\end{array}$ & Axial & $3 / 1$ & $3300 / 60$ & $260 \times 260$ & $128 \times 96$ & \\
\hline T1W FS VIBE MRA & Coronal & $5 / 1$ & $3.85 / 1.42$ & $260 \times 260$ & $256 \times 180$ & 70 \\
\hline T1W FS VIBE pre & Axial & $5 / 1$ & $3.85 / 1.42$ & $260 \times 260$ & $256 \times 180$ & 100 \\
\hline T1W FS VIBE post dynamic $\times 3$ & Axial & $5 / 1$ & $3.85 / 1.42$ & $260 \times 260$ & $256 \times 180$ & $2.5 / 10 / 20$ \\
\hline T1W FS VIBE post whole pelvis & Axial & $5 / 1$ & $3.85 / 1.42$ & $260 \times 260$ & $256 \times 180$ & 70 \\
\hline
\end{tabular}

$A D C$ apparent diffusion coefficient, DWI diffusion weighted imaging, FOV field of view, FS fat saturated, MRA magnetic resonance angiography, TE echo time, TR repetition time, TSE turbo spin echo, VIBE volumetric interpolated breath-hold examination 
b1500, b2000, and b2500 diffusion-weighted images; apparent diffusion coefficient (ADC) map; axial T1 precontrast fat saturated volumetric interpolated breathhold examination (VIBE) images; coronal T1-precontrast fat-saturated MR angiographic VIBE images of the pelvis; serial dynamic axial T1 pre-contrast fat saturated VIBE images obtained after intravenous gadolinium contrast injection (Gadavist $0.1 \mathrm{mmol} / \mathrm{kg}$; Bayer Healthcare Pharmaceuticals, Wayne, NJ, USA); axial fat-saturated T1-weighted delayed postcontrast VIBE images.

Two diagnostic radiologists with 12 and 6 years of experience in interpreting multiparametric prostate MR imaging, respectively, independently reviewed the MR imaging exams before and after PAE. The radiologists used DynaCAD software (InVivo, Philips Healthcare, Amsterdam, Netherlands) on two separate workstations to perform semiautomated prostatic volumetric measurements of the prostate using the MR T2-weighted images. Prostatic volumes were manually confirmed by calculation of the ellipsoid volume formula $(\mathrm{L} \times \mathrm{W} \times \mathrm{H} \times \pi / 6)$. Discordant measurements were resolved by consensus agreement. The diagnostic radiologists also reviewed MR imaging before and after PAE for any prostate gland lesions suspicious for clinically significant cancer according to version 2.1 of Prostate Imaging-Reporting and Data System (PI-RADS) [29].

\section{Prostate artery embolization}

All PAE procedures were performed by a single operator with thirteen years of angiographic and embolization experience and four years of experience performing PAE. All patients received one dose of ciprofloxacin $400 \mathrm{mg}$ administered intravenously for infection prophylaxis. All procedures were performed under moderate (conscious) sedation with local anesthesia in a therapeutic angiography unit. A unilateral left trans-radial arterial approach was utilized in all patients. Real time ultrasound was used to visualize patency and access of the left radial artery, which entered with a micropuncture set, 21-gauge needle, and a 5-French (-F) sheath. Patients underwent digital subtraction angiography (DSA) and PAE with a digital flat-panel detector fluoroscopy system (Innova $4100 \mathrm{IQ}$; General Electric Healthcare, Chicago, IL, USA) with nonionic intravenous contrast (Omnipaque $350 \mathrm{mg} / \mathrm{mL}$; General Electric Healthcare, Chicago, IL, USA). Internal iliac arterial angiography in the ipsilateral oblique projection was performed to identify the right and left prostatic arteries, accessory prostatic arteries, and variant anatomy. Pelvic and prostatic arteries were catheterized using a combination of wires and catheters: 5-F Cobra 2 catheter (Cook Medical, Bloomington, IN, USA), 4-F Berenstein catheter (Merit Medical Systems,
Incorporated, South Jordan, UT, USA), 2.4-F Progreat microcatheter (Terumo Interventional Systems, Tokyo, Japan) and 0.014-inch Transend microguide wire (Stryker Neurovascular, Fremont, CA, USA). The prostatic arteries were identified with DSA. Advanced imaging was performed with localized intraoperative cone-beam computed tomography $(\mathrm{CT})$ with intravenous contrast (100 mL Isovue 370, Bracco Diagnostics, Milan, Italy) prior to embolization. Cone-beam CT images were transmitted, reconstructed in three dimensions, and reviewed to confirm prostatic arterial vascular anatomy, prostate gland vascular supply, and ensure the absence of vascular supply to adjacent anatomical structures, such as the urinary bladder, penis, and rectum. Bilateral PAE was then performed to stasis with a primary embolic agent: 100$250 \mu \mathrm{m}$ Embospheres (Merit Medical Systems, Incorporated, South Jordan, UT, USA) and a secondary embolic agent: $2 \mathrm{~mm}$ and $3 \mathrm{~mm}$ CX coils (Boston Scientific, Marlborough, MA, USA). A band compression device was utilized to achieve radial arterial vascular access closure in all patients.

DSA, which was performed with a flat panel detector fluoroscopy system, and cone-beam CT both utilize photoelectric $x$-rays with ionizing radiation to visualize, identify, and catheterize the prostatic arteries during PAE. In accordance with the American College of Radiology, European Union of Radiology, and Society of Interventional Radiology, ionizing radiation was used in a fashion as low as reasonably achievable (ALARA) to minimize exposure of patients to radiation [30]. Low radiation doses to patients were maintained and further reduced with intraprocedural cone-beam $\mathrm{CT}$ in combination with three dimensional reconstruction that in turn abbreviated procedure times [30].

The Quality Improvement Guidelines for Percutaneous Transcatheter Embolization were used to classify complications after PAE; major complications are complications that require inpatient treatment and/ or surgery and minor complications are complications that can be treated with conservative and/or outpatient treatment [31].

\section{Statistical analysis}

The clinical metrics of IPSS, QoL, Qmax, PVR, PV, and PSA were expressed as quantitative values with means and standard deviations (SD). These quantitative values were analyzed with a Wilcoxon signed rank test using SAS software, version 9.4 (SAS Institute Incorporated, Cary, NC, USA). A probability value of $P<0.05$ or lower was considered statistically significant. We had no missing information for the patients and data that were presented in this study. 


\section{Results}

72 patients with GPH were included with a mean baseline prostate volume of $303 \mathrm{~mL}$ (range 201-644 mL). Mean patient age was 71 years old (range $53-89$ years); 72 males; 34 patients identified as European descent, 21 patients identified as African descent, 12 patients identified as Asian descent, and 5 patients identified with various descents.

Bilateral PAE was technically successful in all patients (Fig. 1: representative patient). Clinical metrics before PAE and 12 months and 24 months after PAE were collected (Table 2). Clinical metrics for each patient were retrieved from the EMR. Mean IPSS decreased from

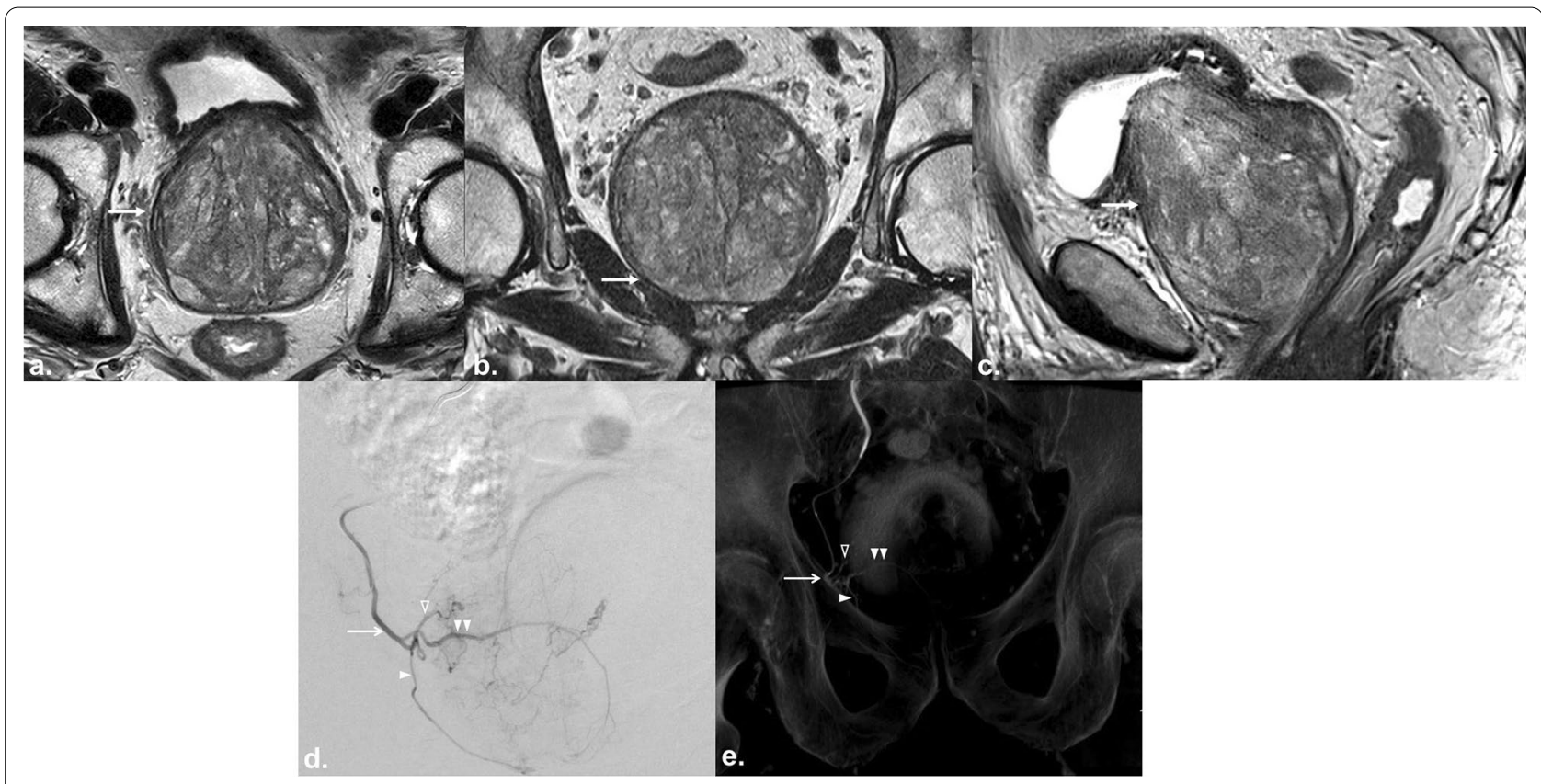

Fig. 1 A 71 year-old patient with severe lower urinary tract symptoms (LUTS) from giant prostatic hyperplasia (GPH) underwent prostate artery embolization (PAE). a Axial T2-weighted turbo spin echo (TSE) image, b coronal T2-weighted TSE image, and $\mathbf{c}$ sagittal T2-weighted image from multiparametric magnetic resonance (MR) imaging show giant hyperplasia of the prostate gland (arrow) that measures $312 \mathrm{~mL}$ in volume. $\mathbf{d}$ Digital subtraction angiography (DSA) of selective catheterization of the right internal iliac artery anterior division shows a common origin of the right prostatic artery (straight arrow), which is hypertrophied, and the right superior vesical artery (open arrowhead). The anterior/lateral prostatic artery (single arrowhead) and the posterior/lateral prostatic artery (double arrowheads) are hypertrophied with a corkscrew pattern of the intraprostatic arterioles. e Cone-beam computed tomography (CT) with intravenous contrast in the coronal plane after selective catheterization of the internal iliac artery anterior division shows the anatomy of the right prostatic artery: a common origin of the right prostatic artery (straight arrow) and the right superior vesical artery (open arrowhead), hypertrophy of the anterior/lateral prostatic artery (single arrowhead) and the posterior/lateral prostatic artery (double arrowheads), and no vascular supply to the adjacent anatomical structures, to include the urinary bladder, penis, and rectum

Table 2 Clinical metrics summary

\begin{tabular}{lccc}
\hline Clinical metric & $\begin{array}{c}\text { Before PAE } \\
\text { Mean } \pm \text { SD }\end{array}$ & $\begin{array}{l}\text { 12 months after PAE } \\
\text { Mean } \pm \text { SD }\end{array}$ & $\begin{array}{c}\text { 24 months after PAE } \\
\text { Mean } \pm \text { SD }\end{array}$ \\
\hline IPSS (points) & $26.5 \pm 5.0$ & $18.0 \pm 4.5$ & $10.0 \pm 4.0$ \\
QoL (points) & $6.0 \pm 1.0$ & $4.0 \pm 1.0$ & $2.0 \pm 1.0$ \\
Qmax (mL/sec) & $8.0 \pm 2.0$ & $14.0 \pm 5.0$ & $18.0 \pm 4.0$ \\
PVR (mL) & $198 \pm 20.0$ & $152 \pm 25.0$ & $90 \pm 15.0$ \\
PV (mL) & $303 \pm 20.0$ & $258 \pm 15.0$ & $209 \pm 15.0$ \\
PSA (ng/mL) & $11.2 \pm 2.5$ & $9.5 \pm 1.5$ & $<.9 \pm 1.5$ \\
\hline
\end{tabular}

IPSS International Prostate Symptom Score, $m L$ milliliter, PAE prostate artery embolization, $P S A$ prostate specific antigen, $P V$ prostate volume, $P V R$ postvoid residual volume, $Q$ max peak flow rate, $Q o L$ urinary quality of life, $S D$ standard deviation 
$26.5 \pm 5.0$ (SD) to $18.0 \pm 4.5 \quad(P<0.0 .01) 12$ months after PAE and decreased from $18.0 \pm 4.5$ to $10.5 \pm 4.0$ $(P<0.01) 24$ months after PAE. Mean QoL decreased from $6.0 \pm 1.0$ to $4.0 \pm 1.0(P<0.01) 12$ months after PAE and decreased from $4.0 \pm 1.0$ to $2.0 \pm 1.0(P<0.01)$ 24 months after PAE. Mean Qmax increased from $8.0 \mathrm{~mL} / \mathrm{s} \pm 2.0 \mathrm{~mL} / \mathrm{s}$ to $14 \mathrm{~mL} / \mathrm{s} \pm 5.0 \mathrm{~mL} / \mathrm{s}(P<0.01)$ at 12 months to $18 \mathrm{~mL} / \mathrm{s} \pm 4.0 \mathrm{~mL} / \mathrm{s}(P<0.01)$ at 24 months. Mean PVR decreased from $198.0 \mathrm{~mL} \pm 20.0 \mathrm{~mL}$ to $152.0 \mathrm{~mL} \pm 25.0 \mathrm{~mL}(P<0.01)$ at 12 months and decreased from $152.0 \mathrm{~mL} \pm 25.0 \mathrm{~mL}$ to $90 \mathrm{~mL} \pm 15.0 \mathrm{~mL}$ $(P<0.01)$ at 24 months. Mean PV decreased from $303.0 \mathrm{~mL} \pm 20.0 \mathrm{~mL}$ to $258.0 \mathrm{~mL} \pm 15.0 \mathrm{~mL}(P<0.01)$ at 12 months and decreased from $258.0 \mathrm{~mL} \pm 15.0 \mathrm{~mL}$ to $209.0 \mathrm{~mL} \pm 15.0 \mathrm{~mL}(P<0.01)$ at 24 months. Mean PSA decreased from $11.2 \mathrm{ng} / \mathrm{mL} \pm 2.5 \mathrm{ng} / \mathrm{mL}$ to $9.5 \mathrm{ng} /$ $\mathrm{mL} \pm 1.5 \mathrm{ng} / \mathrm{mL}(P<0.05)$ at 12 months to $7.9 \mathrm{ng} /$ $\mathrm{mL} \pm 1.5 \mathrm{ng} / \mathrm{mL}(P<0.05)$ at 24 months.

Minor complications occurred in 55 (76\%) patients, resolved between two and seven days after PAE, and were conservatively managed. 20 (28\%) patients experienced acute urinary retention that required temporary urinary bladder catheterization, 14 (19\%) patients experienced urethral pain and dysuria, 11 (15\%) patients experienced hematuria, eight (11\%) patients experienced hemospermia, and two (3\%) patients experienced small hematomas at the radial arterial access site. No major complications occurred.

No lesion suspicious for clinically significant cancer was detected on multiparametric MR imaging obtained in patients before or after PAE. All patients had PI-RADS v2.1 scores of PI-RADS 1: very low (clinically significant cancer is highly unlikely to be present) and PI-RADS 2 : low (clinically significant cancer is unlikely to be present); no patient had scores of PI-RADS 3: intermediate (the presence of clinically significant cancer is equivocal), PIRADS 4: high (clinically significant cancer is likely to be present), or PI-RADS 5: very high (clinically significant cancer is highly likely to be present).

\section{Discussion}

Patients with LUTS due to prostatic hyperplasia that do not respond to medical therapy may be candidates for surgical and minimally invasive treatments. However patients with GPH have limited treatment options due to risks associated with these therapies. Simple prostatectomy, which is the gold standard, and minimally invasive urological procedures may have complications such as blood loss that requires transfusion, urinary incontinence, urinary bladder neck stenosis, and urethral strictures [5-8]. Moreover these surgical and minimally invasive treatments require general anesthesia, which has its own associated risks.
The current set of studies on the efficacy of PAE for treatment of LUTS from prostatic hyperplasia continues to grow. Our study can be added to the contemporary data. This investigation demonstrated the long term efficacy of PAE for treatment of severe LUTS from GPH: prostate volumes greater than $200 \mathrm{~mL}$, in a large patient cohort at 12 months and 24 months after PAE. Mean value for IPSS, QoL, PVR, PV, and PSA significantly decreased and mean Qmax significantly increased at 12 months and at 24 months after PAE. The patients in our investigation continued to experience significant reductions in QoL, Qmax, PVR, and PSA after 12 months and at 24 months after PAE that may be potentially attributed to sustained long term hormonal downregulation and diminished neuromuscular feedback as well as greater initial prostate volumes (greater than $200 \mathrm{~mL}$ ). These long term results are similar to prior investigations that illustrated the long term efficacy of PAE for treatment of LUTS due to prostate hyperplasia with prostate volumes $80-100 \mathrm{~mL}$, in contrast to this investigation, which examined PAE treatment of LUTS from GPH with prostate volumes greater than $200 \mathrm{~mL}$ [5-13]. These studies demonstrated significant decreased severity of symptoms, improved quality of life, increased peak flow rates, and reduction in post void residual and prostate volumes and PSA levels [5-13]. Prior investigators have shown the efficacy of PAE in patients with LUTS from large prostatic hyperplasia: prostate volume greater than $100 \mathrm{~mL}$ however clinical outcomes specifically in patients with GPH, prostate volumes greater than $200 \mathrm{~mL}$, have not been reported [5-13]. Bhatia et al. reported a single patient whom underwent PAE for treatment of severe LUTS from GPH had significant symptomatic relief at three months: IPSS decreased from 26.0 to 4.0, QoL decreased from 6.0 to 1.0, and PV decreased from 571 to $270 \mathrm{~mL}$; PVR was not measured before PAE due to inability to void and was $50 \mathrm{~mL}$ after PAE.[27]. Mathevosian et al. showed similar success and short term efficacy in eight patients whom underwent PAE for severe LUTS from GPH and had sustained symptomatic relief at eight months: IPSS decreased from 20.5 to 3.8, QoL decreased from 4.4 to 1.4 , and PV decreased from 318 to $212 \mathrm{~mL}$ [28]. However the limitations of these studies are small patient cohorts, one and eight, and short follow-up times, 3 months and 8 months $[27,28]$. Larger cohorts of patients and long term efficacy have not been examined. Moreover an upper limit prostate volume for the efficacy of PAE has not been established. This upper limit volume number can be used to match patients to the appropriate therapy.

We confirmed that PAE is not only an effective treatment but also a safe therapy for severe LUTS in patients with GPH (prostate volumes greater than 
$200 \mathrm{~mL}$ ) because no major complications occurred in 72 patients. No patient experienced a major complication, which required inpatient treatment and/or surgery. $76 \%$ of patients experienced at least one minor complication, which was either conservatively managed, treated in an outpatient or ambulatory fashion, or was self-limited and resolved. 20 (28\%) patients experienced acute urinary retention that required temporary urinary bladder catheterization that was likely due to anticipated increased pressure and compression of the intraprostatic urethra from swelling, edema, and ischemic necrosis of the prostate gland after PAE. The urethral pain and dysuria in 14 (19\%) patients, the hematuria in $11(15 \%)$ patients, and the hematospermia in eight $(11 \%)$ patients may have been caused by non-target embolization of the urethra and/ or penis or passage of blood from ischemic necrosis of the prostate gland.

Our study has objective strengths with limitations. While we examined the largest number of patients whom underwent treatment of LUTS from GPH with PAE to date, results may be improved with larger cohorts. The length of follow-up time was longer than any other prior study: 24 months, which establishes sustainability, nevertheless longer-term follow-up studies may verify sustained patient outcomes. Our patients were referred from urologists and therefore subject to intrinsic referral bias. This bias was abated by blinding the interpreting radiologists to the indication for the multiparametric MR imaging exams. There were additional limitations to this investigation. This study was a single-institution, retrospective experience at a tertiary care academic medical center with urology, PAE, and MR availability and experience. Therefore our results may not be transferrable to all patient populations due to accessibility and contraindications. While no major complications occurred in 72 patients, this investigation was retrospective and therefore prospective safety may not be transitively established. PAE is a procedure that may not be widely available to all patients. Certain patients with LUTS from GPH may not be candidates for PAE. If a patient has a contraindication to receiving procedural anesthetic moderate sedation, then a patient cannot undergo PAE. Certain patients may have contraindications to undergo MR imaging, such as medical devices, hardware, or claustrophobia, for prostate volume measurement. In these patients, CT may be used for follow-up imaging for prostate volume measurement. Future research may be directed to prospective, multivariate comparisons of the efficacy of minimally invasive urological procedures and PAE in large patient cohorts for long followup times.

\section{Conclusions}

PAE is an efficacious and safe treatment that provides significant and sustained relief of severe LUTS in patients with GPH. PAE may play an important role in the treatment patients with severe LUTS from GPH in whom medical therapy has failed, are not candidates for surgical and minimally invasive treatments, or refuse surgical treatment. We hope that these results, along with future investigations directed to prospective, multivariate, comparative and randomized studies, may provide patients with the best treatment to optimize symptom relief, minimize morbidity, and achieve safe and successful outcomes.

\section{Abbreviations}

GPH: Giant prostatic hyperplasia; BPH: Benign prostatic hyperplasia; PAE: Prostatic artery embolization; LUTS: Lower urinary tract symptoms; IPSS: International Prostate Symptom Score; mL: Milliliter; PV: Prostate volume; PVR: Postvoid residual volume; QoL: QoL urinary quality of life; Qmax: Peak flow rate; PSA: Prostate specific antigen; MRI: Magnetic resonance imaging; ADC: Apparent diffusion coefficient; DWI: Diffusion weighted imaging; FOV: Field of view; FS: Fat saturated; MRA: Magnetic resonance angiography; TE: Echo time; TR: Repetition time; TSE:Turbo spin echo; VIBE: Volumetric interpolated breath-hold examination; F: French; DSA: Digital subtraction angiography; IRB: Institutional review board; EMR: Electronic medical records; HIPAA: Health Information Portability and Accountability Act.

\section{Acknowledgements \\ None.}

\section{Authors' contributions}

ASS developed the concept and design, performed critical revision of the manuscript for intellectual content, performed imaging interpretations, acquired data, which was analyzed and interpreted, completed statistical analysis, supervised the investigation, and performed final approval. SM performed critical revision of the manuscript, performed imaging interpretations, and acquired data, which was analyzed and interpreted. LMF performed critical revision of the manuscript, performed imaging interpretations, and acquired data, which was analyzed and interpreted. MGN performed critical revision of the manuscript, performed imaging interpretations, and acquired data, which was analyzed and interpreted. AS performed critical revision of the manuscript, performed imaging interpretations, and acquired data, which was analyzed and interpreted. VSK performed critical revision of the manuscript, performed imaging interpretations, and acquired data, which was analyzed and interpreted. All authors read and approved the final manuscript.

\section{Authors' information}

A.S.S. is a board-certified, fellowship-trained radiologist. He is an Assistant Professor of Radiology in the Department of Diagnostic, Molecular, and Interventional Radiology at the Icahn School of Medicine at Mount Sinai in New York City, New York. His areas of clinical expertise are multiparametric MR imaging of the prostate and multivisceral transplants. His research has resulted in numerous exhibits at national and international conferences as well as several peer-reviewed publications and a textbook chapter. S.M. is a board-certified, fellowship-trained radiologist. He is the Director of MR imaging and CT at Valley Radiology Associates in San Jose, California. His research has resulted in multiple educational and scientific exhibits, peer-reviewed publication, and textbook chapters. L.M.F. is a board-certified, fellowship-trained radiologist. She is an Assistant Professor of Radiology in the Department of Radiology at New York Presbyterian/Weill Cornell Medicine. Dr. Flisnik's clinical expertise is in multi-parametric MR imaging of the prostate and PET-CT oncological stag ing and treatment response. Her research has resulted in exhibits at national and international conferences. She is a reviewer for a peer-reviewed journal. M. G. N. is a board-eligible physician in diagnostic radiology. Dr. Nellamattathil completes his residency in Diagnostic Radiology at MedStar Georgetown 
University Hospital in 2021, where he was elected Chief Resident. He will complete his fellowship in Body Imaging at Northwestern Memorial Hospital in 2021. A.S. is a board-certified, fellowship-trained interventional radiologist at Beverly Radiology Medical Group in Los Angeles, California. Dr. Sharma's clinical expertise is in minimally invasive vascular interventions and interventional oncology. Dr. Sharma completed his fellowship in Interventional Radiology at Brigham and Women's Hospital in 2020. His research has resulted in numerous educational and scientific exhibits and peer-reviewed publications. V.S.K. is a board-certified, fellowship-trained radiologist. He currently works as an Associate Professor of Clinical Radiology in the Department of Radiology at the University of Texas Health Science Center San Antonio where he is the Director of Abdominal Imaging. He is academically very successful and presents scientific papers, posters, and educational exhibits at national radiology meetings. He is actively involved in teaching medical students, radiology residents, and abdominal radiology fellows and conducts clinical research.

\section{Funding}

None.

\section{Availability of data and materials}

The data sets used and analyzed during the current study are available from the corresponding author on reasonable request.

\section{Ethics approval and consent to participate}

This study was performed in compliance with the 1996 Health Information Portability and Accountability Act (HIPAA). The investigation location and source of the participants in this investigation was Mount Sinai Hospital in New York, New York where Institutional Review Board and Ethics Committee approved the study (IRB-19-02489), granted ethical approval, and all patients provided written informed consent to allow their medical records to be examined and used in this retroprospective study. A standardized research protocol for the data collection was utilized.

\section{Consent for publication}

Written informed consent was obtained from the patients and any accompanying images. Patients gave consent for their personal or clinical details along with any identifying images to be published in this study. A copy of the written consent is available for review by the Editor-in-Chief of this journal.

\section{Competing interests}

The authors declare that they have no competing interests.

\section{Author details}

${ }^{1}$ Department of Diagnostic, Molecular, and Interventional Radiology, Icahn School of Medicine At Mount Sinai, 1000 10th Avenue, New York, NY 10019, USA. ${ }^{2}$ Valley Radiology Medical Associates, San Jose, CA, USA. ${ }^{3}$ Department of Radiology, New York Presbyterian/Weill Cornell Medical Center, New York, NY, USA. ${ }^{4}$ Department of Radiology, MedStar Georgetown University Hospital, Washington, DC, USA. ${ }^{5}$ Department of Interventional Radiology, Brigham and Women's Hospital, Boston, MA, USA. ${ }^{6}$ Department of Radiology, University of Texas Health Science Center San Antonio, San Antonio, TX, USA.

Received: 10 May 2020 Accepted: 24 September 2020 Published online: 08 October 2020

\section{References}

1. American Urological Association Clinical Guidelines. Surgical management of lower urinary tract symptoms attributed to benign prostatic hyperplasia. 2018. https://www.auanet.org/guidelines/benign-prost atic-hyperplasia/lower-urinary-tract-symptoms (2018).

2. Oelke M, Bachmann A, Descazeaud A, Emberton M, Gravas S, Michel MC, et al. European association of urology: EAU guidelines on the treatment and follow-up of non-neurogenic male lower urinary tract symptoms including benign prostatic obstruction. Eur Urol. 2013;64:118-40.

3. Lourenco T, Pickard R, Vale L, Grant A, Fraser C, MacLennan G, et al. Benign prostatic enlargement team: minimally invasive treatments for benign prostatic enlargement: systematic review of randomised controlled trials. BMJ. 2008;337:a1662.
4. McWilliams JP, Bilhim TA, Carnevale FC, et al. Society of Interventional Radiology Multisociety Consensus Position Statement on Prostatic Artery Embolization for Treatment of Lower Urinary Tract Symptoms Attributed to Benign Prostatic Hyperplasia: From the Society of Interventional Radiology, the Cardiovascular and Interventional Radiological Society of Europe, Société Française de Radiologie, and the British Society of Interventional Radiology: Endorsed by the Asia Pacific Society of Cardiovascular and Interventional Radiology, Canadian Association for Interventional Radiology, Chinese College of Interventionalists, Interventional Radiology Society of Australasia, Japanese Society of Interventional Radiology, and Korean Society of Interventional Radiology. J Vasc Interv Radiol. 2019;30(5):627-37.

5. Pisco J, Bilhim T, Pinheiro LC, et al. Prostate embolization as an alternative to open surgery in patients with large prostate and moderate to severe lower urinary tract symptoms. J Vasc Interv Radiol. 2016;27:700-8.

6. de Assis AM, Moreira AM, de Paula Rodriguez VC, et al. Prostatic artery embolization for treatment of benign prostatic hyperplasia in patients with prostates greater than 90 grams: a prospective single center study. J Vasc Interv Radiol. 2015;26:87-93.

7. Kurbatov D, Giorgio IR, Lepetukhin A, et al. Prostatic artery embolization for prostate volume greater than $80 \mathrm{~cm}^{3}$ : results from a single center prospective study. Urology. 2014;84:400-4.

8. Pisco JM, Bilhim T, Pinheiro LC, et al. Medium and long term outcome on prostate artery embolization for patients with benign prostatic hyperplasia: results in 630 patients. J Vasc Interv Radiol. 2016;27:1115-22.

9. Bagla S, Smirniotopoulos JB, Orlando JC, et al. Comparative analysis of prostate volume as a predictor of outcome in prostate artery embolization. J Vasc Interv Radiol. 2015;26:1832-8.

10. Uflacker A, Haskal ZJ, Bilhim T, et al. Meta-analysis of prostatic artery embolization for benign prostatic hyperplasia. J Vasc Interv Radiol. 2016;27:1686-97.

11. Wang MQ, Guo LP, Zhang GD, et al. Prostatic arterial embolization for the treatment of lower urinary tract symptoms due to large $(>80 \mathrm{ml})$ benign prostatic hyperplasia: results of midterm follow-up from Chinese population. BMC Urol. 2015;15:33.

12. Bhatia S, Sinha VK, Harward S, et al. Prostate artery embolization in patients with prostate volumes of $80 \mathrm{~mL}$ or more: a single institution retrospective experience of 93 patients. J Vasc Interv Radiol. 2018;29:1392-8.

13. Bhatia S, Sinha VK, Kava B, et al. Efficacy of prostatic artery embolization for catheter-dependent patients with large prostate sizes and high comorbidity scores. J Vasc Interv Radiol. 2018;29:78-84.

14. Ashamalla GR, Ahmed AM. Giant prostatic hypertrophy. Arch Surg. 1972;105:769-70.

15. Fishman JR, Merrill DC. A case of giant prostatic hyperplasia. Urology. 1993:42:336-7.

16. De Silva-Gutiérrez A, Pérez-Evia CA, Alcocer-Gaxiola B, Martínez-Méndez ME. Giant prostatic hyperplasia. A case report and literature review. Rev Mex Urol. 2010;70:183-6.

17. Wang L, Davis P, McMillan K. A case of giant prostatic hyperplasia. Asian J Urol. 2016;3(1):53-5.

18. Domínguez A, Gual J, Muñoz-Rodríguez J, et al. Giant prostatic hyperplasia: case report of $3987 \mathrm{~mL}$. Urology. 2016;88:e3-4.

19. Aghamir SMK, Khatami F, Rahimi MR, Guitynavard F. Giant benign prostatic hyperplasia: a case report. Urol Case Rep. 2019;28:101051.

20. Lacy JM, Bole R, Hendrix L, Strup S. Retropubic prostatectomy for giant benign prostatic hyperplasia. Can J Urol. 2015;22(5):8000-2.

21. Ogawa $S$, Manome M, Yabe $M$, et al. A giant prostatic hyperplasia treated by open surgery. Int J Gen Med. 2012;5:1009-12.

22. Zeng QS, Zhao YB, Wang BQ, et al. Minimally invasive simple prostatectomy for a case of giant benign prostatic hyperplasia. Asian J Androl. 2017;19(6):717-8.

23. Learney RM, Malde S, Downes M, Shrotri N. Successful minimally invasive management of a case of giant prostatic hypertrophy associated with recurrent nephrogenic adenoma of the prostate. BMC Urol. 2013;13:18.

24. Sevryukov FA, Nakagawa K, Kochkin AD, et al. A case of successful plasma transurethral enucleation of benign prostatic hyperplasia the size of 530 $\mathrm{cm}^{3}$. Urologiia. 2019;2:59-63.

25. Glybochko PV, Rapoport LM, Enikeev ME, Enikeev DV. Holmium laser enucleation of the prostate (HoLEP) for small, large and giant prostatic hyperplasia: tips and tricks. Urologia. 2017;84(3):169-73. 
26. Enikeev DV, Glybochko PV, Alyaev YG, et al. Holmium laser enucleation of the prostate (HOLEP) for small, large and giant prostatic hyperplasia. Practice guidelines. Experience of more than 450 surgeries. Urologiia. 2016:4:63-9.

27. Bhatia S, Kava B, Pereira K, et al. Prostate artery embolization for giant prostatic hyperplasia. J Vasc Interv Radiol. 2015;26(10):1583-5.

28. Mathevosian S, Plotnik AN, McWilliams JP. Prostate artery embolization for giant prostatic enlargement: short-term efficacy and safety. J Vasc Interv Radiol. 2019;30(11):1820-3.

29. Weinreb JC, Barentsz JO, Choyke PL, et al. PI-RADS Prostate imagingreporting and data system: 2015, Version 2. Eur Urol. 2016;69:16-40.

30. Schott P, Katoh M, Fischer N, Freyhardt P. Radiation dose in prostatic artery embolization using cone-beam CT and 3D roadmap software. J Vasc Interv Radiol. 2019;30(9):1452-8.
31. Angle JF, Siddiqi NH, Wallace MJ, Kundu S, Stokes L, Wojak JC, et al. Quality improvement guidelines for percutaneous transcatheter embolization: Society of Interventional Radiology Standards of Practice Committee. J Vasc Interv Radiol. 2010;21:1479-86.

\section{Publisher's Note}

Springer Nature remains neutral with regard to jurisdictional claims in published maps and institutional affiliations.
Ready to submit your research? Choose BMC and benefit from:

- fast, convenient online submission

- thorough peer review by experienced researchers in your field

- rapid publication on acceptance

- support for research data, including large and complex data types

- gold Open Access which fosters wider collaboration and increased citations

- maximum visibility for your research: over $100 \mathrm{M}$ website views per year

At BMC, research is always in progress.

Learn more biomedcentral.com/submissions 